\title{
L'encadrement des stagiaires en milieu de travail
} Étude exploratoire dans un cadre formel d'alternance en formation professionnelle initiale

\section{Supervising interns in the workplace}

An exploratory study in the context of a formal Co-operative Education program for initial profession training

\section{La dirección de los practicantes en el medio de trabajo} Un estudio exploratorio en el marco formal de alternancia al
inicio de la formación profesional

\section{Élisabeth Mazalon, Claudia Gagnon et Sandra Roy}

Volume 42, numéro 1, printemps 2014

L'alternance en formation : nouveaux enjeux, autres regards?

URI : https://id.erudit.org/iderudit/1024568ar

DOI : https://doi.org/10.7202/1024568ar

Aller au sommaire du numéro

\section{Éditeur(s)}

Association canadienne d'éducation de langue française

ISSN

1916-8659 (numérique)

Découvrir la revue

Citer cet article

Mazalon, É., Gagnon, C. \& Roy, S. (2014). L'encadrement des stagiaires en milieu de travail : étude exploratoire dans un cadre formel d'alternance en formation professionnelle initiale. Éducation et francophonie, 42(1), 113-135.

https://doi.org/10.7202/1024568ar
Résumé de l'article

En prenant une part plus active dans la formation des futurs travailleurs, la personne responsable de l'encadrement des élèves en milieu de travail dans un contexte de formation professionnelle initiale, le superviseur en entreprise (MELS, 2006) ou le tuteur (Agulhon et Lechaux, 1996), exerce différentes responsabilités auprès de l'élève qui vont au-delà de ses tâches professionnelles visant la production. Quelles sont ces tâches? Quel est le type d'encadrement offert dans les entreprises québécoises impliquées dans les projets d'alternance en formation professionnelle initiale? Cet article vise à rendre compte des résultats de l'enquête par questionnaires menée auprès de plus d'une centaine de superviseurs en entreprises dans différentes régions du Québec. Sur le plan organisationnel, les résultats montrent que nous sommes essentiellement en présence d'une alternance concertée (Mazalon et Bourassa, 2003), les types Individuel supervisé et Collectif supervisé étant ressortis comme les plus fréquents. Sur le plan de la pédagogie ou de la formation en entreprise, à proprement parler, les résultats révèlent la présence d'un guidage de l'activité (Savoyant, 1995) centré sur l'exécution et le contrôle.
Tous droits réservés @ Association canadienne d'éducation de langue française, Ce document est protégé par la loi sur le droit d'auteur. L’utilisation des 2014 services d'Érudit (y compris la reproduction) est assujettie à sa politique d'utilisation que vous pouvez consulter en ligne.

https://apropos.erudit.org/fr/usagers/politique-dutilisation/ 


\title{
L'encadrement des stagiaires en milieu de travail Étude exploratoire dans un cadre formel d'alternance en formation professionnelle initiale
}

\author{
Élisabeth MAZALON \\ Université de Sherbrooke, Québec, Canada \\ Claudia GAGNON \\ Université de Sherbrooke, Québec, Canada
}

\section{Sandra ROY}

Université de Sherbrooke, Québec, Canada

\section{RÉSUMÉ}

En prenant une part plus active dans la formation des futurs travailleurs, la personne responsable de l'encadrement des élèves en milieu de travail dans un contexte de formation professionnelle initiale, le superviseur en entreprise (MELS, 2006) ou le tuteur (Agulhon et Lechaux, 1996), exerce différentes responsabilités auprès de l'élève qui vont au-delà de ses tâches professionnelles visant la production. Quelles sont ces tâches? Quel est le type d'encadrement offert dans les entreprises québécoises impliquées dans les projets d'alternance en formation professionnelle initiale? 
Cet article vise à rendre compte des résultats de l'enquête par questionnaires ${ }^{1}$ menée auprès de plus d'une centaine de superviseurs en entreprises dans différentes régions du Québec. Sur le plan organisationnel, les résultats montrent que nous sommes essentiellement en présence d'une alternance concertée (Mazalon et Bourassa, 2003), les types Individuel supervisé et Collectif supervisé étant ressortis comme les plus fréquents. Sur le plan de la pédagogie ou de la formation en entreprise, à proprement parler, les résultats révèlent la présence d'un guidage de l'activité (Savoyant, 1995) centré sur l'exécution et le contrôle.

\section{ABSTRACT}

Supervising interns in the workplace: an exploratory study in the context of a formal Co-operative Education program for initial profession training

Élisabeth MAZALON

University of Sherbrooke, Québec, Canada

Claudia GAGNON

University of Sherbrooke, Québec, Canada

Sandra ROY

University of Sherbrooke, Québec, Canada

In taking a more active role in the training of future workers, those responsible for supervising students in the workplace in the context of initial professional training, business supervisors (MELS, 2006) or tutors (Agulhon and Lechaux, 1996), exercise a variety of responsibilities with the student besides professional duties targeting production. What are these duties? What type of supervision is offered at Québec companies involved in Co-operative Education projects for initial professional training? This article reports the results of a questionnaire ${ }^{1}$ conducted among over one hundred business supervisors in various regions of Québec. On the organizational level, the results essentially reveal a coordinated Co-operative Education process (Mazalon and Bourassa, 2003), with individual and group supervision emerging most frequently. Strictly speaking, on the pedagogical level or for corporate training, the results show that supervisors act mainly as coaches (Savoyant, 1995), with a focus on performance and control.

1. Ce projet de recherche a été subventionné par le Comité national de l'alternance travail-études (CNATE) soutenu financièrement par le ministère de l'Éducation, du Loisir et du Sport (MELS, 2010-2011).

1. This research project was funded by the Comité national de l'alternance travail-études (CNATE), financially supported by the ministère de l'Éducation, du Loisir et du Sport (MELS, 2010-2011). 


\section{RESUMEN}

\section{La dirección de los practicantes en el medio de trabajo: un estudio exploratorio en el marco formal de alternancia al inicio de la formación profesional}

Élisabeth MAZALON

Universidad de Sherbrooke, Quebec, Canadá

Claudia GAGNON

Universidad de Sherbrooke, Quebec, Canadá

Sandra ROY

Universidad de Sherbrooke, Quebec, Canadá

Al tomar una parte más activa en la formación de los futuros trabajadores, la persona responsable de la dirección de los alumnos en el medio de trabajo en un contexto de formación inicial, el supervisor en empresa (MELS, 2006) o el tutor (Agulhon et Lechaux, 1996), ejerce diferentes responsabilidades sobre el alumno que van mas allá de su tarea profesional concerniente a la producción. ¿Cuáles son esas tareas? ¿Cuál es el tipo de dirección que ofrecen las empresas quebequenses implicadas en los proyectos de alternancia al iniciarse la formación profesional? Este artículo describe los resultados de una encuesta por cuestionario ${ }^{1}$ realizada entre más de una centena de supervisores en empresa en las diferentes regiones de Quebec. En el aspecto organizacional, los resultados muestran que estamos, esencialmente, en presencia de una alternancia concertada (Mazalon et Bourassa, 2003), los tipos individual y colectivo supervisados aparecen como los más frecuentes. En el aspecto pedagógico o de la formación en empresa, propiamente dicho, los resultados muestran la presencia de un encausamiento de la actividad (Savoyant, 1995) centrado en la ejecución y el control.

\section{Problématique}

Au cours des dernières années, les recherches québécoises se sont intéressées plus particulièrement au stage ou à la dimension du travail dans l'alternance en formation professionnelle et technique, et ce, en matière de sens ou d'apprentissages pour les élèves (Mazalon, Beaucher et Langlois, 2010; Hardy et Ménard; 2008; Landry,

1. Este proyecto de investigación fue subvencionado por el Centro nacional de alternancia trabajo-estudios (CNATE), apoyado financieramente por el ministerio de Educación, Recreación y Deporte (MELS, 2010-2011) 
Bouchard et Pelletier, 2002), de même qu'en matière d'encadrement des stagiaires (Hardy, Bouteiller et Parent, 2000; Hardy et Ménard, 2002; Hardy et Parent, 2000). Gagnon (2008a) a quant à elle consacré sa recherche doctorale aux pratiques éducatives qu'adoptent des enseignants et des formateurs en entreprise en contexte d'alternance. Bien que la recherche de Gagnon ait permis de lever en partie le voile entourant les pratiques de formateurs ${ }^{2}$ en entreprise et de préciser certaines pratiques relatives au guidage de l'activité (Savoyant, 1995), il importe de souligner que les résultats concernent uniquement un secteur de formation, l'agriculture, et ont été obtenus à partir d'entrevues et d'observations auprès d'un nombre restreint de répondants. En somme, à la suite de Hardy et Parent (2000), il nous apparaît que ces recherches ont permis «de dissiper, partiellement, le brouillard qui entoure trop fréquemment les pratiques tutorales en entreprise» (p. 252), mais plusieurs éléments demeurent encore flous et restent à préciser.

Dans cette perspective, en nous appuyant sur les résultats de ces recherches, nous avons cru important d'analyser les pratiques d'encadrement chez des superviseurs $^{3}$ en entreprise participant aux projets d'alternance en formation professionnelle dans différents secteurs de formation.

\section{Contexte général et objectifs de recherche}

Les programmes d'études en formation professionnelle initiale (FP) ${ }^{4}$ intègrent tous, à l'exception du secteur de la construction, un stage d'intégration obligatoire de trois semaines ou plus crédité pour la diplomation. Cependant, la mise en œuvre de l'alternance se fait sous la responsabilité des administrateurs scolaires ou en fonction de l'initiative des acteurs pédagogiques, principalement les enseignants. Le développement de l'alternance offerte sous l'appellation «alternance travail-études (ATE)» au Québec est soutenu par des subventions de démarrage et de fonctionnement par le ministère de l'Éducation, du Loisir et du Sport (MELS). Sur le plan éducatif, le guide pédagogique destiné aux intervenants du milieu scolaire appuie une stratégie éducative qui vise clairement une alternance intégrative centrée sur la conformité des apprentissages avec les objectifs du programme d'études, tout en adoptant une approche pédagogique où l'élève devrait être l'acteur central de sa formation (MELS,

2. Dans le cadre de sa thèse, l'auteure privilégie le terme formateur en entreprise pour nommer la personne responsable de l'encadrement en entreprise.

3. Le terme superviseur a été privilégié dans cette recherche afin de respecter la notion définie dans le guide pédagogique du MELS (2006) et dans le guide pratique du superviseur de stage en entreprise, diffusé par l'Association québécoise alternance études-travail. Voir le site de l'Association à l'adresse http://www.aqaet.qc.ca/portail/index.aspx?sect=0\&module=5\&module2=1\&MenulD=960\&CPage=1

4. Les programmes d'études menant à un diplôme d'études professionnelles (DEP) s'adressent aux personnes titulaires d'un diplôme d'études secondaires ou l'équivalent, aux personnes ayant atteint l'âge de 16 ans au 30 septembre de l'année scolaire où débute leur formation professionnelle, aux personnes ayant atteint l'âge de 18 ans et possédant les préalables fonctionnels prescrits pour le programme ou aux personnes ayant obtenu les unités de la troisième secondaire et qui poursuivront, en concomitance avec leur formation professionnelle, leur formation générale du second cycle du secondaire (MELS, 2009). Ces programmes diffèrent du Parcours de formation axée sur l'emploi (PFAE) traité par Charbonneau, Samson et Rousseau dans l'article précédent, qui permet l'apprentissage d'un métier semi-spécialisé et s'adresse aux élèves du deuxième cycle du secondaire (plus de 15 ans) présentant diverses difficultés scolaires. 
2006). Par cette orientation, le MELS tient à bien marquer la différence entre, d'une part, les finalités et les modalités organisationnelles et pédagogiques des séquences en entreprise dans un projet d'ATE et, d'autre part, celles des stages d'intégration et des stages cliniques dans les programmes de santé. Concrètement, les projets subventionnés en FP doivent comporter un minimum de deux activités de formation en milieu de travail, dont le contenu est dicté par l'école et qui représentent au moins $20 \%$ de la totalité des heures du programme d'études. Ces activités, dont l'intention pédagogique est le développement partiel ou total de compétences, sont soumises à l'évaluation sommative des modules de formation concernés et donnent droit à des unités. Le stagiaire a un statut d'élève et, de ce fait, il ne reçoit pas de rémunération.

Toutefois, comme nous l'avons déjà souligné (Mazalon et Bourassa, 2003), il faut différencier ce qui est de l'ordre institutionnel et organisationnel de la pratique des acteurs engagés dans les projets. Afin de mieux comprendre la réalité quant à l'encadrement des stagiaires en entreprise, un mandat a été confié au Collectif de recherche sur la formation professionnelle (CRFP) de l'Université de Sherbrooke pour réaliser une étude dans le cadre des projets en alternance travail-études mis en œuvre en formation professionnelle initiale.

\section{Cadre d'analyse de l'encadrement des stagiaires en milieu de travail}

Plusieurs auteurs ont traité de la formation et de l'encadrement en entreprise, que ce soit dans un contexte d'alternance ou dans un contexte d'apprentissage en milieu de travail. Les écrits anglo-saxons s'attardent plus précisément à l'apprentissage en milieu de travail et abordent entre autres les profils de participation et d'apprentissage (Fuller et Unwin, 2003, 2004), le système d'activité et apprentissage en milieu de travail (Engeström, 2001), la typologie de l'expérience de travail (Guile et Griffiths, 2001), les pratiques de participation et de formation en milieu de travail (Billett, 2003, 2004; Billett, Barker et Hernon-Tinning, 2004). Les recherches sur l'alternance ou par apprentissage en formation professionnelle initiale dans un contexte francophone traitent plus particulièrement des acteurs et de leurs rôles respectifs (Hardy et Ménard, 2002; Mazalon et Bourassa, 2003) et des pratiques des tuteurs ou des formateurs en entreprise (Agulhon et Lechaux, 1996; Pasquier, 1998; Kunégel, 2005; Gagnon 2008a; Filliettaz, 2012) pour ne nommer que ceux-ci. La prochaine section présente les différents principes organisationnels et pédagogiques retenus dans notre recherche pour opérationnaliser la notion d'encadrement du superviseur en entreprise.

\section{L'encadrement d'un point de vue organisationnel et pédagogique}

Si dans le langage courant la notion de supervision est surtout associée au domaine de l'enseignement au Québec, le terme encadrement est privilégié dans les entreprises pour spécifier la fonction du superviseur en entreprise. Sur le plan théorique, Gagnon (2008a) en parlant de pratique d'encadrement englobe la planification 
des tâches de l'alternant (phase préactive), la supervision au quotidien des apprentissages et du travail de celui-ci (phase interactive) et l'évaluation (phase postactive). En s'appuyant sur les phases de prise en charge définies par Pasquier (1998), Hardy et Parent (2000), à partir des perceptions de quinze tuteurs en techniques d'usinage et en modelage, décrivent le processus de formation pendant les stages en entreprise en cinq phases : la sélection, l'accueil, l'observation, le suivi et l'évaluation. Quant au terme utilisé pour désigner la personne responsable de l'encadrement en entreprise, les auteurs empruntent le titre de «tuteur», largement exploité dans les recherches européennes, ainsi que la notion de tutorat pour la fonction du tuteur. Selon la typologie présentée par Le Douaron (1993) et reprise par d'autres (Agulhon et Lechaux, 1996; Pasquier, 1998) deux types de tuteurs sont distingués: les tuteurs "opérationnels ", qui assurent eux-mêmes l'encadrement du stagiaire dont ils sont responsables, et les tuteurs " hiérarchiques » qui assument la responsabilité administrative du stage, délèguent une partie de leur responsabilité d'encadrement à un subordonné avec qui le stagiaire travaille, et discutent avec cette personne des tâches qui sont les plus appropriées pour le stagiaire. Pour Agulhon et Lechaux (1996), «le tuteur est [...] un adulte de référence qui guide et soutient le jeune dans sa progression" (p. 23). Pour ces auteurs, les tuteurs s'inscrivent dans une logique d'organisation des apprentissages, de gestion de la mise au travail, d'intégration des normes de la production ou d'accueil formel. Quant à Hardy et Ménard (2002), elles empruntent la typologie de l'expérience de travail de Guile et Griffiths (2001) pour examiner les formes d'encadrement offertes aux élèves par les enseignants et les tuteurs. Les auteurs relèvent «une concentration des perceptions et des comportements correspondant au modèle traditionnel» (p. 6). L'étude montre également que l'encadrement de l'expérience de travail est caractérisé par plusieurs étapes : la préparation du stage par les enseignants ou un coordonnateur, c'est-à-dire la sélection d'élèves dans certains cas, l'explication des objectifs et du déroulement, l'assignation de tâches diversifiées, le conseil sur le plan technique par les tuteurs secondés par des employés pendant le stage, et la visite des enseignants ou du coordonnateur au cours du stage afin d'évaluer les habiletés pratiques de l'élève ou de discuter des tâches réalisées et du travail du stagiaire avec le tuteur. Dans une perspective didactique, pour Mayen (1999), la première démarche du tuteur sera de trouver des situations d'apprentissage pour le tutoré, qu'il appelle des «situations potentielles de développement». À la suite de Savoyant (1995), qui s'inscrit dans la théorie de l'activité, Gagnon (2008a) a analysé les pratiques des formateurs en entreprise - pour ce qui est de la phase interactive ou de la supervision au quotidien - sous l'angle du guidage de l'activité. Bien qu'il ne s'agisse pas d'un processus linéaire obligé, le guidage de l'activité comprend, entre les opérations d'exécution et les opérations d'orientation, des opérations de contrôle de l'exécution où le formateur en entreprise communément nommé superviseur dans notre recherche effectue ou devrait effectuer une observation plus ou moins continue de ce que fait le stagiaire et des interventions visant à corriger l'exécution par le stagiaire en cas de «non-conformité». Enfin, les opérations d'orientation "correspondent à ce que l'on peut appeler la part intelligente de l'action, c'est-à-dire à l'identification de la situation et à la prise de décision» (Mayen, 
1999, p. 25). À cet égard, il ne s'agit pas simplement de nommer ou «de définir des règles d'action, mais de dégager les connaissances qui les fondent» (Savoyant, 1995, p. 97).

Dans le cadre de l'alternance travail-études en FP au Québec, le superviseur en entreprise est décrit comme un personnage clé dans la réussite des activités en milieu de travail qui doit « développer sa vision de la formation en alternance travailétudes; créer un environnement propice à l'apprentissage; accompagner l'élève en milieu de travail; participer à l'évaluation formative de l'élève» (MELS, 2006, p. 14). Sur le plan organisationnel, les enseignants du programme d'études concerné ont la responsabilité d'établir les modalités organisationnelles des stages, stages perlés ou hebdomadaires, d'une durée de deux à six semaines réparties sur l'année scolaire avec l'obligation d'un retour en classe après chaque stage. Il revient aussi à l'établissement scolaire d'élaborer les documents d'accompagnement du stage (cahier du superviseur en entreprise et cahier du stagiaire). Les enseignants conçoivent également les canevas pédagogiques requis pour l'évaluation des séquences de formation en entreprise qui relèvent exclusivement du centre de formation dans le cas des séquences qui visent le développement de compétences. Il est demandé au superviseur d'accueillir l'élève dans le milieu de travail, de superviser le travail et les apprentissages de l'élève et de participer à l'évaluation de l'élève. Il revient donc à l'enseignant responsable des stages d'informer le superviseur en entreprise de son rôle et de ses responsabilités ainsi que des attentes du centre de formation au regard des objectifs poursuivis par les stages.

\section{Méthodologie}

Un questionnaire a été élaboré à partir des objectifs spécifiques de la recherche définis en collaboration avec le Comité national de l'alternance travail-études (CNATE). L'un des objectifs de ce comité était de compiler des données empiriques sur l'implication des entreprises en tant qu'espace de formation dans le cadre de la formation professionnelle initiale en alternance afin d'être en mesure d'analyser l'état de situation du dossier et de diagnostiquer les problèmes. Dans le cadre de cette recherche, les chercheurs ont dû composer avec des exigences d'ordre financier et temporel. Considérant le budget initial, les échéanciers serrés (une année) et la grande difficulté de recueillir des listes d'employeurs auprès des responsables des projets d'ATE, la mise en ligne d'un questionnaire, pour compléter les questionnaires téléphoniques, administrés au moyen d'une interface Web, a été privilégiée afin de rejoindre en peu de temps le plus grand nombre possible de participants. Afin de respecter une durée de passation raisonnable, le questionnaire téléphonique initial a été allégé tout en conservant les mêmes grandes catégories et les mêmes questions. 
À la suite d'une préanalyse des questionnaires, des sous-énoncés ont été supprimés et des questions avec échelle de Lickert ont été ajoutées ${ }^{5}$.

S'inspirant des cadres utilisés dans la recherche de Gagnon (2008a) et de Mazalon (1998), des questions ont été bâties en vue de prendre en compte des items relatifs aux objets de formation (tâches en entreprises / compétences visées), des items processuels (dynamique de la communication / relation, rôles du superviseur et de l'élève, processus de guidage de l'activité) et des items relatifs aux éléments organisationnels (outils utilisés, organisation du temps et de l'espace). Le principal but de cette enquête exploratoire était de recueillir de l'information factuelle et de dresser un portrait des différents thèmes abordés à l'aide d'une analyse descriptive des données excluant les analyses d'association et de corrélation étant donné le peu de participants dans certains secteurs de formation. Les données recueillies correspondent aux pratiques d'encadrement "déclarées" par les superviseurs ou employeurs en entreprise, pratiques qui peuvent, dans certains cas, être différentes des «pratiques réelles» (Gagnon, 2008a et b). Toutefois, ce choix théorique qui s'appuie sur le courant de l'interactionnisme symbolique permet de comprendre ce qui se fait en amont des gestes effectifs et, finalement, de construire des représentations de ce qui est fait. Dans ce sens, les auteures tiennent à souligner qu'il s'agit là d'un avancement considérable dans la connaissance de «la part cachée» de l'alternance travail-études dans un contexte de formation professionnelle initiale au Québec, d'autant plus que la recherche a permis de rejoindre des entreprises dans des secteurs diversifiés, aux quatre coins du Québec.

\section{Définition de l'échantillon et déroulement de l'enquête}

Conformément au devis soumis par l'équipe de recherche, la population visée était les employeurs ou les superviseurs des entreprises engagées dans les stages en ATE dans des projets d'alternance existant depuis plus de trois ans. À la suite de trois relances auprès des personnes responsables dans les commissions scolaires, une liste de 1130 employeurs a été établie ${ }^{6}$. De ce nombre, 137 personnes de différentes entreprises ont répondu, complètement ou partiellement, au questionnaire en ligne ou par téléphone. Le taux de réponse global au questionnaire est donc de 23,25\%. Le secteur le plus représenté reste celui de l'administration, commerce et informatique (39\%), alors que le taux de participation pour les autres secteurs varie entre $0 \%$ et 13 $\%$ (voir le tableau 1). Pour l'année 2007-2008, ce secteur est en effet celui qui est le plus représenté, avec $23,5 \%$ de projets totaux en ATE, suivi des secteurs agriculture et pêche (16\%), entretien d'équipement motorisé (12\%), alimentation et tourisme ainsi que fabrication mécanique (10,5\%). Les autres secteurs représentent moins de

5. Le questionnaire comportait 52 questions regroupées en 6 sections: 1) la description de l'entreprise, 2) le partenariat entreprise et milieu scolaire, 3) les pratiques d'encadrement, 4) la connaissance du soutien offert et de certains programmes, 5) le statut du superviseur, 6) les conditions facilitantes et les obstacles quant à I'implication dans l'entreprise dans la formation d'un stagiaire en ATE. Selon la nature des questions, celles-ci offraient la possibilité de répondre par oui ou par non ou par quatre choix de réponse (0- jamais; 1 - parfois; 2- souvent; 3- tout le temps).

6. La liste comptait 1130 entreprises réparties seulement dans 10 secteurs de formation sur les 19 secteurs admissibles à l'ATE, regroupant 29 programmes de formation différents et relevant de 18 commissions scolaires. De cette liste, 589 entreprises avec des adresses courriel ont pu être recensées. 
$5 \%$ des projets totaux d'ATE seulement. Par ailleurs, il est possible que le médium utilisé, de type questionnaire en ligne, convienne mieux pour certains secteurs, notamment celui de l'administration, commerce et informatique, que pour d'autres.

\section{Tableau 1. Répartition des répondants selon le secteur de formation}

\begin{tabular}{|c|c|c|c|}
\hline & $\begin{array}{l}\text { Total des répondants } \\
\text { Nombre (n) } \\
\text { et pourcentage (\%) }\end{array}$ & $\begin{array}{l}\text { Total des répondants } \\
\text { Nombre }(n) \\
\text { et pourcentage }(\%)\end{array}$ & $\begin{array}{l}\text { Total des répondants } \\
\text { Nombre }(\mathrm{n}) \\
\text { et pourcentage }(\%)\end{array}$ \\
\hline $\begin{array}{r}\text { Ensemble } \\
\text { Secteurs de formation }\end{array}$ & $\begin{array}{l}137 \\
(100,0 \%)\end{array}$ & $\begin{array}{l}137 \\
(100,0 \%)\end{array}$ & $\begin{array}{l}137 \\
(100,0 \%)\end{array}$ \\
\hline Administration, commerce et informatique & $53(38,7 \%)$ & $53(38,7 \%)$ & $53(38,7 \%)$ \\
\hline Agriculture et pêche & $8(5,8 \%)$ & $8(5,8 \%)$ & $8(5,8 \%)$ \\
\hline Alimentation et tourisme & $18(13,1 \%)$ & $18(13,1 \%)$ & $18(13,1 \%)$ \\
\hline Arts & - & - & - \\
\hline Bois et matériaux connexes & - & - & - \\
\hline Bâtiment et travaux publics & - & - & - \\
\hline Environnement et aménagement du territoire & - & - & - \\
\hline Électrotechnique & - & - & - \\
\hline Entretien d'équipement motorisé & $8(5,8 \%)$ & $8(5,8 \%)$ & $8(5,8 \%)$ \\
\hline Fabrication mécanique & $9(6,6 \%)$ & $9(6,6 \%)$ & $9(6,6 \%)$ \\
\hline Foresterie et papier & $13(9,5 \%)$ & $13(9,5 \%)$ & $13(9,5 \%)$ \\
\hline Communication et documentation & - & - & - \\
\hline Mécanique d'entretien & $11(8 \%)$ & $11(8 \%)$ & $11(8 \%)$ \\
\hline Mines et travaux de chantier & - & - & - \\
\hline Métallurgie & $10(7,3 \%)$ & $10(7,3 \%)$ & $10(7,3 \%)$ \\
\hline Transport & $2(1,5 \%)$ & $2(1,5 \%)$ & $2(1,5 \%)$ \\
\hline Cuir, textile et habillement & - & - & - \\
\hline Santé & $1(0,7 \%)$ & $1(0,7 \%)$ & $1(0,7 \%)$ \\
\hline Soin esthétique & $4(2,9 \%)$ & $4(2,9 \%)$ & $4(2,9 \%)$ \\
\hline
\end{tabular}

\section{Caractéristiques des répondants}

Quand on examine la répartition des répondants selon leurs caractéristiques personnelles (tableau 2), on remarque que la majorité d'entre eux ont le statut de superviseur ou d'employeur superviseur $(77 \%)$. Ces répondants sont pour plus des deux tiers des hommes (69,3\%), ils ont plus de 40 ans (75\%), comptent plus de dix ans d'ancienneté dans l'entreprise (57\%) et presque la moitié (47\%) d'entre eux ont plus de cinq ans d'expérience en encadrement de stagiaires. 
Tableau 2. Répartition des répondants (questionnaire téléphonique et en ligne) selon les caractéristiques personnelles

\begin{tabular}{|c|c|c|c|}
\hline & $\begin{array}{l}\text { Répondants au } \\
\text { questionnaire en } \\
\text { ligne } \\
\text { Nombre }(n) \\
\text { et pourcentage }(\%)\end{array}$ & $\begin{array}{l}\text { Répondants au } \\
\text { questionnaire en } \\
\text { ligne } \\
\text { Nombre }(n) \\
\text { et pourcentage }(\%)\end{array}$ & $\begin{array}{l}\text { Répondants au } \\
\text { questionnaire en } \\
\text { ligne } \\
\text { Nombre }(n) \\
\text { et pourcentage }(\%)\end{array}$ \\
\hline Ensemble & $\begin{array}{l}89 \\
(100,0 \%)\end{array}$ & $\begin{array}{l}48 \\
(100,0 \%)\end{array}$ & $\begin{array}{l}137 \\
(100,0 \%)\end{array}$ \\
\hline Statut & $72(100,0 \%)$ & $48(100,0 \%)$ & $120(100,0 \%)$ \\
\hline Superviseur & $26(36,1 \%)$ & $24(50,0 \%)$ & $50(41,7 \%)$ \\
\hline Employeur ou RH (sans supervision directe) & $13(18,1 \%)$ & $15(31,3 \%)$ & $28(23,3 \%)$ \\
\hline Employeur superviseur & $33(45,8 \%)$ & $9(18,8 \%)$ & $42(35,0 \%)$ \\
\hline$\hat{A} g e$ & $48(100,0 \%)$ & $47(100,0 \%)$ & $95(100,0 \%)$ \\
\hline Entre 20 et 30 ans & $3(6,3 \%)$ & $4(8,5 \%)$ & $7(7,4 \%)$ \\
\hline Entre 31 et 40 ans & $7(14,6 \%)$ & $10(21,3 \%)$ & $17(17,9 \%)$ \\
\hline Entre 41 et 50 ans & $18(37,5 \%)$ & $19(40,4 \%)$ & $37(38,9 \%)$ \\
\hline Plus de 50 ans & $20(41,7 \%)$ & $14(29,8 \%)$ & $34(35,8 \%)$ \\
\hline Ancienneté dans l'entreprise & $48(100,0 \%)$ & $45(100,0 \%)$ & $93(100,0 \%)$ \\
\hline Moins d'un an & $1(2,1 \%)$ & $0(0,0 \%)$ & $1(1,1 \%)$ \\
\hline De un an à trois ans & $7(14,6 \%)$ & $3(6,7 \%)$ & $10(10,8 \%)$ \\
\hline De quatre ans à dix ans & $9(18,8 \%)$ & $20(44,4 \%)$ & $29(31,2 \%)$ \\
\hline Plus de dix ans & $31(64,6 \%)$ & $22(48,9 \%)$ & $53(57,0 \%)$ \\
\hline Niveau de scolarité & $48(100,0 \%)$ & $45(100,0 \%)$ & $93(100,0 \%)$ \\
\hline Secondaire & $8(16,7 \%)$ & $11(24,5 \%)$ & $19(20,4 \%)$ \\
\hline Diplôme d'études professionnelles & $14(29,2 \%)$ & $9(20,0 \%)$ & $23(24,7 \%)$ \\
\hline Diplôme de niveau collégial & $14(29,2 \%)$ & $15(33,3 \%)$ & $29(31,2 \%)$ \\
\hline Diplôme de niveau universitaire & $12(25,0 \%)$ & $10(22,2 \%)$ & $22(23,7 \%)$ \\
\hline Expérience en supervision de stagiaires & $48(100,0 \%)$ & $48(100,0 \%)$ & $96(100,0 \%)$ \\
\hline Moins d'un an & $4(8,3 \%)$ & $4(8,3 \%)$ & $8(8,3 \%)$ \\
\hline Entre un et deux ans & $6(12,5 \%)$ & $3(6,3 \%)$ & $9(9,4 \%)$ \\
\hline Entre deux et cinq ans & $15(31,3 \%)$ & $19(39,6 \%)$ & $34(35,4 \%)$ \\
\hline Plus de cinq ans & $23(47,9 \%)$ & $22(45,8 \%)$ & $45(46,9 \%)$ \\
\hline $\begin{array}{l}\text { A déjà suivi une formation en lien avec la } \\
\text { supervision de stagiaires ou d'employés }\end{array}$ & $48(100,0 \%)$ & $48(100,0 \%)$ & $96(100,0 \%)$ \\
\hline Non & $30(62,5 \%)$ & $41(85,4 \%)$ & $71(74,0 \%)$ \\
\hline Oui & $18(37,5 \%)$ & $7(14,6 \%)$ & $25(26,0 \%)$ \\
\hline
\end{tabular}

Aussi, la plupart d'entre eux n'ont jamais suivi de formation en lien avec la supervision de stagiaires ( $74 \%)$ et plus de la moitié $(55 \%)$ ont un niveau de scolarité postsecondaire (tableau 2). Sur les $26 \%$ des répondants qui disent avoir reçu une formation en lien avec la supervision des stagiaires, tous ont reçu des formations 
offertes au sein de leur entreprise ou sont titulaires d'un diplôme en administration ou en pédagogie. Aucun des répondants n'a signalé une formation offerte par un centre de formation professionnelle.

\section{Résultats}

Les résultats présentés dans cet article comportent deux parties: une première partie relève plus particulièrement des représentations des participants qui soustendent l'encadrement en lien avec quelques éléments organisationnels. On aborde ici les raisons pour l'entreprise de recevoir des stagiaires et la sélection de ces derniers. Une deuxième partie s'attardera plus particulièrement à l'aspect pédagogique de l'encadrement qui englobe la planification des tâches, la supervision au quotidien des apprentissages et du travail de celui-ci ainsi que l'évaluation du stagiaire.

\section{L'encadrement en entreprise d'un point de vue organisationnel}

Quand on examine la répartition des répondants selon les caractéristiques des entreprises qu'ils représentent, on remarque que la majorité d'entre eux viennent d'une entreprise privée (65\%) comprenant plusieurs départements ou services (76\%) et fonctionnant tout au long de l'année (95\%). La majorité des entreprises signalent la présence d'une personne responsable de la supervision des stagiaires (79\%), ainsi que la présence d'autres services ou personnes participant à l'encadrement des stagiaires (71\%). Il est à noter que plus des deux tiers (68\%) des entreprises interrogées ont plus de quatre ans d'expérience de collaboration avec les centres de formation professionnelle. Les entreprises interrogées reçoivent, outre les stagiaires en ATE, majoritairement des stagiaires dirigés vers eux par des centres de formation professionnelle, puisque $61 \%$ disent accueillir des stagiaires pour des stages d'intégration, et près du tiers des entreprises reçoivent des stagiaires du cégep et de l'université (tableau 3). 
Tableau 3. Provenance des stagiaires

\begin{tabular}{|l|r|}
\cline { 2 - 2 } \multicolumn{1}{c|}{} & $\%$ \\
\hline Ensemble & 100,0 \\
\hline Reçoit des stagiaires du cégep & 100,0 \\
Non & 82,2 \\
Oui & 17,7 \\
\hline Reçoit des stagiaires de l'université & 100,0 \\
Non & 86,7 \\
Oui & 13,3 \\
\hline Reçoit des stagiaires d'un programme d'insertion à l'emploi (Emploi-Québec) & 100,0 \\
Non & 94,7 \\
Oui & 5,3 \\
\hline Reçoit des stagiaires de la formation professionnelle & \\
(stage d'intégration de fin de formation) & 100,0 \\
Non & 38,9 \\
Oui & 61,1 \\
\hline Reçoit des stagiaires d'un programme d'apprentissage en milieu de travail & 100,0 \\
Non & 90,3 \\
Oui & 9,7 \\
\hline
\end{tabular}

\section{Raisons pour l'entreprise de recevoir des stagiaires}

De façon générale, les répondants sont plutôt d'accord pour dire qu'ils reçoivent des stagiaires pour les raisons suivantes: permettre à l'étudiant d'avoir une expérience de travail (97\%); contribuer à la formation professionnelle de l'élève (96\%); participer à la formation d'une main-d'œuvre compétente dans le secteur (89\%); sélectionner et recruter des employés (74\%). D’un autre côté, les répondants sont plutôt neutres relativement au fait de recevoir des stagiaires pour rendre service à quelqu'un et ils se montrent plutôt en désaccord avec le fait de recevoir des stagiaires pour remplacer des employés réguliers absents $(80 \%)$. Ces résultats viennent nuancer ceux de Caron et Payeur (2002) qui soulignent dans leur étude que les acteurs du milieu de travail engagés dans l'alternance attribuent prioritairement au stage une valeur positive en relation avec le recrutement de futurs employés. Cependant, les répondants rencontrés dans leur étude étaient des représentants de l'employeur ou du service des ressources humaines ou, encore, des personnes salariées.

Pour compléter les données numériques et pour bien saisir les raisons incitant l'entreprise à s'engager dans des stages, on demandait aux répondants d'écrire ou de dire en quelques mots ce qui motivait l'employeur à recevoir des stagiaires de la formation professionnelle initiale dans son entreprise. Nous avons regroupé les éléments de réponse en cinq catégories: la participation à la formation de la relève, la participation sociale de l'entreprise, le recrutement de la future main-d'œuvre, l'aide supplémentaire et, enfin, l'échange de nouvelles connaissances. Les idées les plus souvent exprimées sont: formation, «former la relève pour assurer une bonne continuité dans nos industries», recrutement, «recruter de futurs candidats pour notre entreprise», aide, «aide dans divers projets en cours» et échange mutuel, "échange des connaissances nouvelles». 
Aussi, sur un total de 140 items issus des réponses d'une centaine de répondants, $48 \%(\mathrm{n}=67)$ étaient consacrés à des raisons liées au besoin de l'entreprise de participer à la formation de la relève. On note deux tendances dans le discours:

- la formation par la pratique et l'expérience de travail: «leur donner l'opportunité de tester leurs connaissances en pratique», «apprendre en milieu de travail, ça leur apporte une expérience de travail significative», «lui permettre de mettre en pratique les connaissances apprises»; et

- le transfert de connaissances de l'expert au stagiaire: «partager l'expérience avec une main-d'œuvre plus expérimentée», "passage des connaissances et acquisition de nouvelles connaissances».

On observe que la deuxième raison avancée pour recevoir des stagiaires, avec un peu moins du quart $(20 \%)$ des énoncés $(n=28)$, est le recrutement de la maind'œuvre. Les répondants parlent de former en prévision de l'embauche ou d'avoir la possibilité d'évaluer un stagiaire à même son stage en prévision de besoins de personnel à combler: "avoir la possibilité de connaître les gens avant de les embaucher», "faire connaître ma profession d'agriculteur et aussi découvrir des employés potentiels».

La troisième raison évoquée pour $16 \%$ des items $(n=22)$ est la participation sociale de l'entreprise. À cet égard, les répondants évoquent leur volonté de faire leur part, de donner une chance aux jeunes: "rendre service au centre de formation professionnelle», "ça fait partie de notre mission sociale, ça nous permet de créer un partenariat avec les établissements scolaires». La quatrième raison mentionnée par les répondants et qui compose $10 \%(\mathrm{n}=14)$ des items de la totalité du discours est l'aide apportée par cette main-d'œuvre supplémentaire: «c'est une façon d'offrir de l'aide à nos techniciens en informatique pour les opérations massives d'installation", "plus de ressources aux tâches de base comme le support téléphonique», "les stagiaires sont une ressource de main-d'œuvre additionnelle lors de la période plus intense pour l'entreprise». Il n'est pas rare que certains répondants associent le besoin de maind'œuvre supplémentaire à celui de partager des connaissances et d'échanger de nouvelles idées: "en faisant un stage, cela leur permet de se faire connaître et de comprendre les rudiments de l'entreprise. De plus, ils apportent des connaissances nouvelles, ce qui permet parfois d'améliorer certaines méthodes». Cette dernière raison est évoquée dans $6 \%(n=9)$ des items qui composent les raisons des répondants à recevoir des stagiaires.

\section{Sélection des stagiaires}

Environ la moitié des entreprises interrogées procèdent à une sélection des stagiaires qu'elles accueilleront (47\%). De ce nombre, plus de la moitié des répondants (68\%) disent favoriser l'entrevue comme mode de sélection et se fier à la recommandation d'un enseignant (69\%). Les résultats scolaires sont très peu considérés dans la sélection des stagiaires (2\%). Quant à l'initiative de recevoir des stagiaires, on note que c'est surtout le milieu scolaire qui est demandeur de service (73\%) et que plus du tiers (42\%) des étudiants sont responsables de la recherche de leur stage. 


\section{L'encadrement d'un point de vue pédagogique ou de formation}

Suivant ce qui a été retenu dans le cadre de référence, les pratiques d'encadrement ont été abordées suivant trois étapes: 1) la préparation du stage ou la planification des tâches; 2) l'accueil et la supervision au quotidien des apprentissages et du travail du stagiaire; 3) l'évaluation de celui-ci.

\section{Préparation du stage}

La majorité des entreprises ont eu une rencontre ou un contact téléphonique préalables avec un enseignant du centre de formation professionnelle $(79 \%)$. Dans la majorité des cas, les répondants mentionnent que l'enseignant a fourni des explications concernant la grille d'évaluation (95\%), les compétences à développer durant le stage (92\%), le rôle du superviseur (88\%), les éléments relatifs à la santé et la sécurité de l'entreprise (SST) (81\%), les modalités pour faciliter l'apprentissage du stagiaire $(73 \%)$, les documents d'information et de formation $(72 \%)$. Pour préciser ces informations, il était demandé aux répondants de se prononcer sur une échelle de 0 (jamais) à 3 (tout le temps), sur la présence de certains éléments au sujet des explications fournies par l'enseignant du CFP. Les explications fournies le plus fréquemment par les enseignants concernent la grille d'évaluation (54\%) et les documents d'information et de formation ( $45 \%$ ). L'élément qui semble retenir le moins l'attention lors de la préparation du stage a trait aux modalités pour faciliter l'apprentissage du stagiaire (23\%) (voir le graphique 1$)$.

Graphique 1. Explications données par l'enseignant lors de la préparation du stage dans l'entreprise (\%)

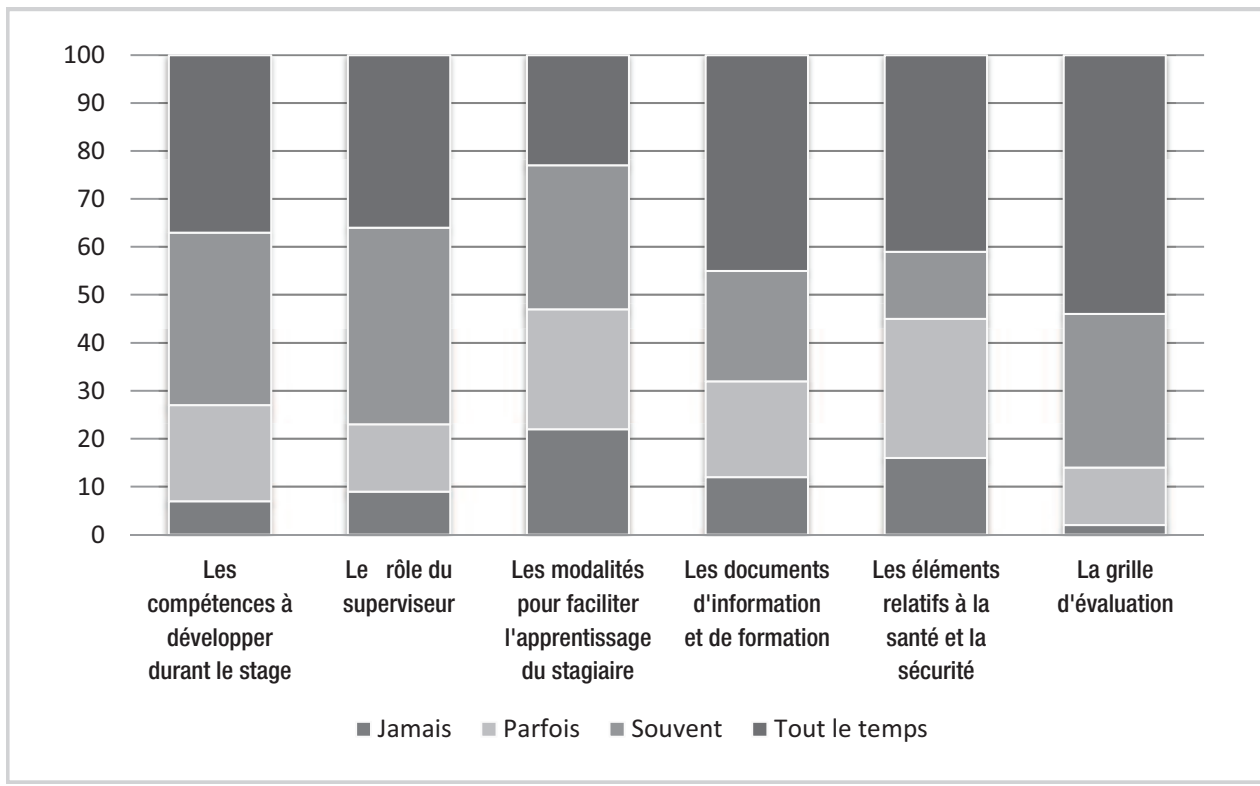




\section{Accueil et supervision au quotidien des apprentissages et du travail}

Dans un contexte d'alternance en formation professionnelle initiale, les élèves sont intégrés aux activités quotidiennes du milieu de travail dans un contexte de production. Dans le cadre de la recherche, il était demandé aux répondants de se prononcer sur la présence de certains éléments au sujet des actions qu'ils faisaient à l'arrivée du stagiaire et durant le stage. Pour l'accueil, on note que les répondants affirment qu'ils effectuent souvent chacune des actions qui leur étaient proposées, c'est-à-dire informer le stagiaire des méthodes de travail (87\%), lui faire visiter l'entreprise $(87 \%)$, l'informer des tâches qu'il devra effectuer (81\%) et lui présenter le personnel (81 \%). C'est l'énoncé «J'ai discuté avec lui de mon rôle de superviseur» qui obtient le plus faible niveau d'accord parmi les actions effectuées à l'arrivée du stagiaire $(66 \%)$.

Quant à l'encadrement du stagiaire, les répondants étaient interrogés sur quatre aspects de l'encadrement: "organisation du temps de travail en présence du stagiaire», "actions posées lors de l'explication des tâches», "actions posées lors de la vérification du travail» et finalement «les actions posées lorsque le stagiaire présente des difficultés».

Les répondants disent effectuer souvent leur travail à peu près normalement (51\%) et confirment avoir souvent et même tout le temps besoin de l'aide des autres employés afin que toutes leurs tâches professionnelles soient faites (83\%). Dans cette perspective, ils disent ne jamais $(70 \%)$ s'occuper uniquement de la supervision des stagiaires. Nous avons regroupé dans les graphiques qui suivent les actions effectuées lors de l'explication des tâches et de la vérification du travail exécuté par le stagiaire, ces items permettant de mieux saisir le guidage de l'activité en entreprise (Savoyant, 1995; Mayen, 1999; Gagnon, 2008a). À cet égard, on remarque que les actions les plus fréquentes correspondent aux opérations d'exécution. Elles consistent à donner des consignes et des directives sur la tâche à effectuer (62\%) et, dans une proportion moins importante, à décrire et à observer la réalisation de la tâche pendant que le stagiaire l'exécute (33\%). On constate par ailleurs que l'utilisation des documents pour expliquer les notions théoriques de la tâche (23\%) et le fait d'expliquer d'autres façons de faire (18\%), qui renvoient aux opérations d'orientation, sont des actions moins fréquentes (graphique 2). 
Graphique 2. Explication des tâches de travail par le superviseur (exécution et orientation) (\%)

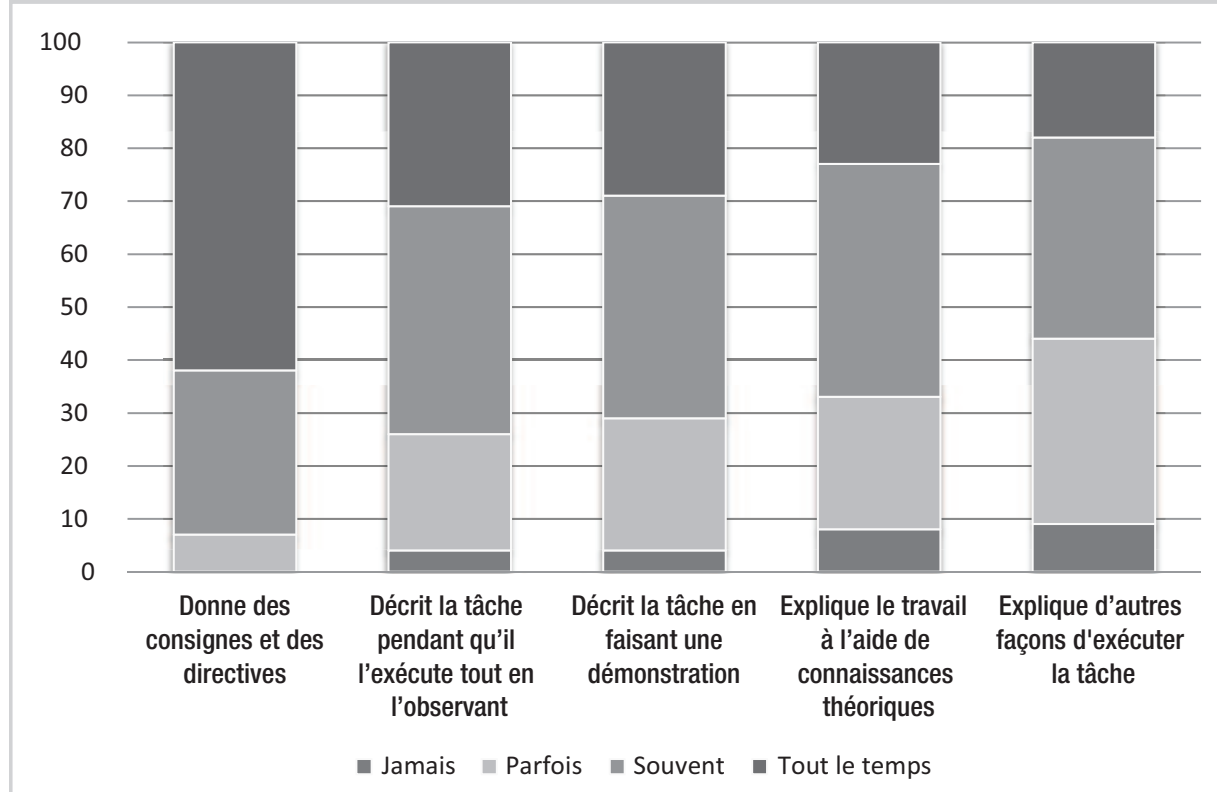

En ce qui a trait aux actions liées à la vérification du travail, qui correspondent aux opérations de contrôle, plus de la moitié des répondants mentionnent corriger tout le temps le stagiaire si le travail n'est pas conforme (57\%) et donner tout le temps une appréciation au stagiaire si le travail est conforme (52\%). Par ailleurs, il est intéressant d'observer que pour plus d'un tiers des participants les opérations de contrôle sont implicites et effectuées tout le temps lors de l'exécution de chaque étape de la tâche (39\%) et par observation et évaluation une fois la tâche terminée (38\%). On note par ailleurs que l'utilisation des documents en lien avec le processus et les résultats des tâches planifiées et qui se réfèrent aux opérations de contrôle est une action moins fréquente (18\%). Par contre, on remarque que l'élément «je ne corrige pas le stagiaire, je refais moi-même le travail mal effectué» est peu fréquent (1\%) (graphique 3).

Pour compléter ces données, on interrogeait aussi les répondants sur leur comportement dans un contexte où le stagiaire présente des difficultés. Cinq types de comportements étaient proposés: rencontre personnellement l'étudiant, tente d'en discuter avec l'enseignant responsable, le laisse poursuivre pour accroître son autonomie, demande à quelqu'un d'autre d'expliquer la tâche, explique et fait une démonstration. Aussi, dans un contexte où l'élève présente des difficultés, on note une tendance plus forte à expliquer à nouveau et à faire une démonstration (46\%), de même qu'une faible propension à en discuter avec l'enseignant responsable (9\%). 
Graphique 3. Vérification du travail effectué par le stagiaire (contrôle) (\%)

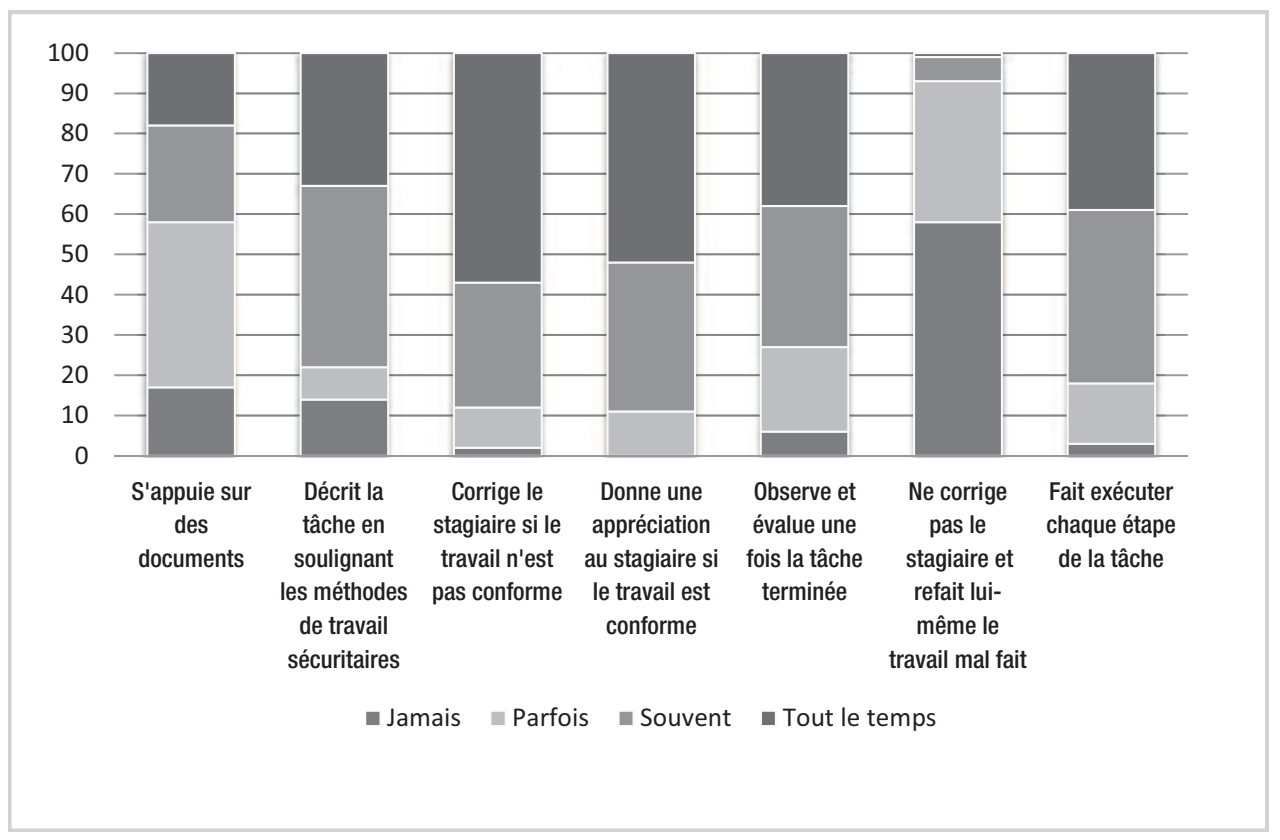

\section{Évaluation du stagiaire}

L'évaluation du stagiaire fait partie intégrante du stage, mais, dans le contexte de l'ATE en formation professionnelle initiale, cette opération reste sous la responsabilité de l'établissement scolaire, étant donné que l'intention pédagogique de la séquence en milieu de travail vise le développement de compétences. Cette évaluation, qui portait sur l'acquisition ou pas des compétences planifiées par l'établissement scolaire, est effectuée par le personnel enseignant, puisque les apprentissages réalisés lors du séjour en entreprise font partie intégrante des programmes d'études et par le fait même sont inclus dans l'évaluation sommative. Selon les modalités prescrites par l'équipe enseignante, l'évaluation peut s'effectuer en milieu de stage ou au retour en classe. Les grilles d'évaluation conçues par le milieu scolaire et remplies par les superviseurs en milieu de travail s'attardent entre autres sur le degré d'exécution des activités professionnelles visées par le centre de formation professionnelle et sur les attitudes et comportements du stagiaire dans le milieu de travail.

Dans le contexte de notre recherche, la grande majorité des répondants disent effectuer une évaluation du stagiaire (93\%). Par contre, il est intéressant de noter que $85 \%$ des répondants utilisent les grilles conçues par le milieu scolaire, dont certaines en partenariat $(10 \%)$, tandis que peu utilisent la grille de l'entreprise $(8 \%)$. On observe qu'environ le tiers des répondants déclarent que l'évaluation est effectuée plusieurs fois au cours du stage (30\%), tandis qu'ils sont un peu plus de la moitié à évaluer uniquement la fin du stage (55\%). On peut noter que les superviseurs en entreprise assument majoritairement leur rôle dans l'évaluation du stagiaire. En 
effet, ils sont une grande majorité à fournir une rétroaction orale à la suite de l'évaluation (90\%). Quant à la rétroaction écrite, elle est aussi présente dans la majorité des cas (90\%) et en présence du stagiaire dans plus de la moitié des situations (56\%). Par contre, il est surprenant que les évaluations soient généralement faites sans la présence de l'enseignant (81\%), étant donné que dans le cadre de l'ATE la détermination du contenu du stage, la supervision et l'évaluation sont la responsabilité première de l'établissement d'enseignement.

En ce qui a trait aux éléments évalués, la grande majorité des répondants mentionnent privilégier tout le temps les compétences à acquérir (84\%) et les critères portant sur l'intérêt et la motivation ( $80 \%$ ) ainsi que sur les attitudes et la persévérance dans les tâches (76\%). D'autres éléments sont également souvent présents dans l'évaluation, mais à plus faible fréquence: le respect des règles de santé et de sécurité (SST) (53\%) et l'adhésion du stagiaire à la culture de l'entreprise (49\%) (graphique 4).

Graphique 4. Éléments d'évaluation privilégiés par le superviseur lors de l'évaluation du stagiaire (\%)

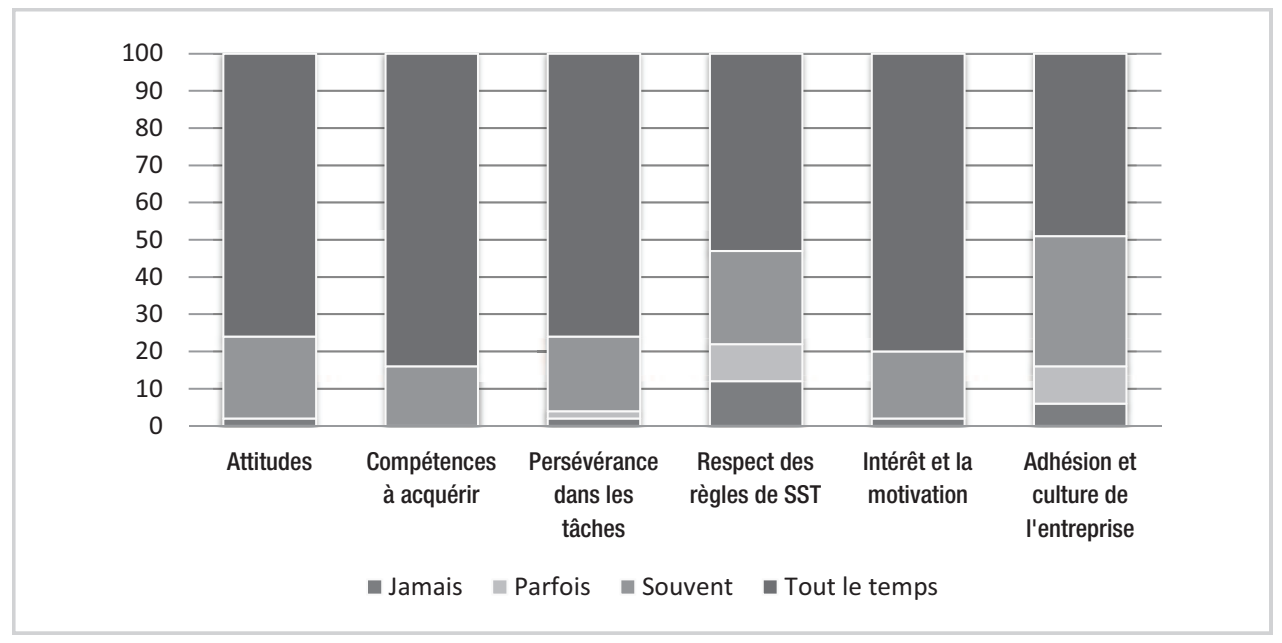

Finalement, les superviseurs avaient à indiquer leur niveau de satisfaction en ce qui concerne certains éléments de l'expérience de supervision de stage. On peut souligner une satisfaction générale à l'égard de l'expérience de supervision de stage. En effet, on remarque que dans l'ensemble les éléments se situent au-delà de $90 \%$, ce qui est très appréciable. Les éléments sur lesquels les répondants se montrent le plus satisfaits se situent sur les plans de leur rôle de superviseur (98\%), de l'implication du stagiaire (98\%), de l'implication de l'entreprise dans l'accueil des stagiaires $(97 \%)$ et de la satisfaction à l'égard du soutien du centre de formation professionnelle $(90 \%)$. 


\section{Discussion}

Les résultats montrent que, même si les pratiques d'encadrement peuvent être différentes, elles s'articulent autour de la volonté de participer à la formation de la relève afin d'assurer une bonne continuité dans les entreprises et de recruter de futurs employés. Les propos recueillis mettent en évidence que les pratiques de supervision sont soutenues par une alternance concertée (Mazalon et Bourassa, 2003) et qu'elles sont marquées par un guidage de l'activité (Savoyant, 1995).

\section{Des pratiques d'encadrement soutenues par une alternance concertée}

Sur le plan organisationnel, les résultats montrent que nous sommes essentiellement en présence d'une alternance concertée, qui se traduit par une structure de formation cohérente entre les deux milieux et dont l'élaboration des activités en entreprise résulte d'un processus plus ou moins consensuel des acteurs concernés (Mazalon et Bourassa, 2003). En effet, l'élève a accès à une personne expérimentée qui connaît, dans la majorité des cas, son plan de formation, qui le soutient dans la réalisation de ses activités de travail et facilite son intégration dans l'entreprise. Plus de la moitié des répondants (69\%) disent favoriser la recommandation d'un enseignant pour sélectionner les stagiaires. L'encadrement des tâches est principalement individuel (dyade). Ce type d'encadrement, nommé par Hardy, Bouteiller et Parent (2000) Individuel supervisé, s'observe dans les milieux de travail où le superviseur est le seul à encadrer le travail du stagiaire, alors que les autres employés n'interviennent qu'occasionnellement. Cependant dans notre recherche, dans la majorité des cas les superviseurs soulignent que l'encadrement se fait aussi dans une équipe de plusieurs employés afin de favoriser la diversification des apprentissages et de maintenir la productivité $(71 \%)$ dans l'entreprise ainsi que, par le fait même, de permettre au stagiaire de recevoir des rétroactions par d'autres membres du personnel. Ce résultat va dans le même sens que les résultats de l'étude menée par Hardy, Bouteiller et Parent (2000), qui définissent ce type Collectif supervisé comme un modèle où superviseur et employés ou collègues de l'équipe ou de l'unité de travail participent à l'encadrement du stagiaire. De façon complémentaire, le superviseur de même que les collègues de travail définissent les tâches et donnent des conseils au stagiaire afin de contribuer à sa formation et à son intégration dans le milieu de travail. Comme nous l'avons déjà souligné (Mazalon, Beaucher et Langlois 2010), il est nécessaire dans tout développement de projet d'alternance de différencier l'alternance organisationnelle de l'alternance pédagogique et de présenter aux partenaires un choix explicite du modèle pédagogique privilégié.

\section{Des pratiques d'encadrement marquées par un guidage de l'activité centré sur l'exécution}

Sur le plan pédagogique ou de la formation en entreprise proprement dite, les résultats révèlent la présence d'un certain guidage de l'activité (Savoyant, 1995) effectué par les superviseurs des entreprises participantes. Les réponses des superviseurs quant aux actions menées auprès des stagiaires suggèrent que le guidage est 
centré essentiellement ou principalement sur les opérations d'exécution, qui consistent à dire "ce qu'il faut faire et non pourquoi il faut le faire" (Savoyant, 1995, p. 93) . En effet, comme nous l'avons vu dans la présentation des résultats, les superviseurs donnent des consignes et des directives sur la tâche à effectuer (62\%) et, dans une proportion moins importante, décrivent et observent la réalisation de la tâche pendant que le stagiaire l'exécute (33\%), ce qui peut renvoyer en partie aux opérations de contrôle. Comme nous l'avons souligné ailleurs (Gagnon, 2008a), bien que la description des opérations d'exécution demeure une activité formative essentielle (Mayen, 1999), il faut souligner qu'il s'agit de la partie la plus évidente et la plus facilement réalisée du guidage (Savoyant, 1996), alors que celui-ci pourrait être fortement enrichi, pour une meilleure formation du stagiaire et un arrimage plus important de la théorie et de la pratique, notamment en intégrant des opérations d'orientation. En outre, comme le souligne Mayen (1999), la description des opérations d'exécution seule est inefficace, car elle pourrait occulter:

- les éléments «non observables» de l'action, c'est-à-dire les informations prises en compte, l'objectif poursuivi, les règles d'action mobilisées, etc.;

- les autres façons d'effectuer l'action;

- les dimensions temporelles de l'action ainsi que la situation de cette action dans une perspective plus large.

Plus largement, ces résultats en ce qui a trait au guidage de l'activité effectué par les superviseurs vont dans le sens d'une conception du stage qui vise l'application et la mise en œuvre de ce qui a été vu préalablement en centre de formation. Le stage n'est pas vu comme un espace d'apprentissage et de développement des compétences, ce qui, on le sait, se situe en porte à faux avec les objectifs de l'alternance en formation professionnelle. Dans ce sens, à notre avis, et comme nous l'avons souligné ailleurs (Gagnon, 2008a), il importe d'outiller davantage les superviseurs en entreprise en matière de guidage de l'activité et d'opérations d'orientation afin que non seulement ils intègrent dans leurs actions auprès des stagiaires le fait d'énoncer ou de définir des règles de fonctionnement, mais également qu'ils dégagent ou permettent aux élèves de «dégager les connaissances qui les fondent» (Savoyant, 1995, p. 97).

Quoi qu'il en soit, il importe de soulever quelques limites au regard de la méthodologie employée pour décrire les pratiques. D’une part, rappelons que ce sont les superviseurs eux-mêmes qui ont porté un regard sur leurs pratiques. Peut-être les résultats auraient-ils été autres si nous avions interrogé les stagiaires. Par ailleurs, l'observation de ces pratiques permettrait sans nul doute d'affiner les résultats de cette étude et de préciser les actions «implicites» et riches d'apprentissages effectuées par les superviseurs auprès des stagiaires. 


\section{Pour conclure...}

Nous soulignons en terminant l'importance du rôle des entreprises dans la formation professionnelle initiale. Dans ce contexte, l'alternance travail-études comme stratégie de formation est un levier essentiel à la qualification de la future main-d'œuvre. Dans ce cas, il est utile de conscientiser et de former les différents intervenants impliqués dans l'alternance, les enseignants et les superviseurs en entreprise, en passant par les directions de centre de formation professionnelle, sur le plein potentiel pédagogique de l'alternance dans le développement professionnel de notre main-d'œuvre. Par ailleurs, nous croyons qu'il est temps de mettre en œuvre, en partenariat avec les milieux professionnels, des ententes de formation et des outils pédagogiques qui tiennent compte des différentes opérations du guidage de l'activité pour favoriser une plus grande ouverture du plan de formation et des grilles d'évaluation afin de créer une dynamique de formation inversée, laquelle partirait de l'entreprise pour aller vers l'école.

\section{Références bibliographiques}

AGULHON, C. (2000). L'alternance: une notion polymorphe, des enjeux et des pratiques segmentées. Revue française de pédagogie, 131 (avril-mai-juin), 55-63.

AGULHON, C. et LECHAUX, P. (1996). Un tutorat ou des tutorats en entreprise. Diversité des pratiques. Recherche et formation, 22, 21-34.

BILLETT, S. (2003). Workplace mentors: Demands and benefits. The Journal of Workplace Learning, 15(3), 105-113.

BILLETT, S. (2004). Workplace participatory practices. Conceptualising workplaces as learning environments. The Journal of Workplace Learning, 16(6), 312-324.

BILLETT, S., BARKER, M. et HERNON-TINNING, B. (2004). Participatory practices at work. Pedagogy, Culture and Society, 12(2), 233-257.

CARON, L. et PAYEUR, C. (2002). Regards contrastés sur les stages et l'alternance dans un cadre régional. Dans C. Landry (dir.), La formation en alternance. État des pratiques et des recherches (p. 309-344). Québec: Presses de l'Université du Québec.

ENGESTROM, Y. (2001). Expansive learning at work: Toward an activity theoretical reconceptualization. Journal of Education and Work, 74(1), 133-156.

FILLIETTAZ, L. (2012). Interactions tutorales et formation des formateurs. Travail et apprentissage, 9, 62-83. 
FULLER, A. et UNWIN, L. (2003). Learning as apprentices in the contemporary UK workplace: Creating and managing expansive and restrictive participation. Journal of Education and Work, 16(4), 407-426.

FULLER, A. et UNWIN, L. (2004). Young people as teachers and learners in the workplace: Challenging the novice-expert dichotomy. International Journal of Training and Development, 8(1), 31-41.

GAGNON, C. (2008a). Arrimage des pratiques éducatives d'enseignants et de formateurs en entreprise en contexte d'alternance. Études de cas en formation professionnelle agricole. Thèse de doctorat en éducation, Université de Sherbrooke, Faculté d'éducation, Sherbrooke.

GAGNON, C. (2008b). Arrimage des pratiques éducatives d'enseignants et de formateurs en entreprises en contexte d'alternance. Études de cas en formation professionnelle agricole. Recherches qualitatives, 27(1), 141-190.

GUILE, D. et GRIFFITHS, T. (2001). Learning through work experience. Journal of Education and Work, 14(1), 113-131.

HARDY, M., BOUTEILLER, D. et PARENT, C. (2000, novembre). Tuteurs et stagiaires en alternance : quelles relations de formation? Communication présentée à la rencontre annuelle du GIRFE, Montréal, Québec.

HARDY, M. et MÉNARD, L. (2002). Marginalité des apprentissages en entreprise : encadrement de la formation professionnelle en alternance par les enseignants et les tuteurs. Texte de communication non publié présenté au Congrès international de PAFIRSE, Formations initiales et continues au regard des recherches et de la philosophie de l'éducation, Pau, 9 au 11 mai 2002.

HARDY, M. et MÉNARD, L. (2008). Alternance travail-études. Les effets de stages dans la formation professionnelle des élèves. Revue des sciences de l'éducation, 34(3), 689-709.

HARDY, M. et PARENT, C. (2000). Perceptions des tuteurs impliqués dans la formation professionnelle initiale en entreprise. Dans D.-G. Tremblay et P. Doray (dir.), Vers de nouveaux modes de formation professionnelle? Rôle des acteurs et des collaborations (p. 247-257). Québec: Presses de l'Université du Québec.

KUNÉGEL, P. (2005). L’apprentissage en entreprise: l'activité de médiation des tuteurs. Éducation permanente, 164(4), 127-138.

LANDRY, C., BOUCHARD, Y. et PELLETIER, C. (2002). Le stage dans l'alternance travail-études au collégial : pour quel rapport au savoir? Dans C. Landry (dir.), La formation en alternance. État des pratiques et des recherches (p. 195-216). Québec: Presses de l'Université du Québec.

LE DOUARON, P. (1993). Alternance et négociation. Éducation permanente, 115, 111-118. 
MAYEN, P. (1999). Des situations potentielles de développement. Éducation permanente, 139, 65-86.

MAZALON, É. (1998). Les représentations des acteurs de l'alternance travail-études: une formation au service de quel partenariat? Dans J.-N. Demol et J.-M. Pilon (dir.), Alternance, développement personnel et local (p. 229-238). Paris : L'Harmattan.

MAZALON, É. et BOURASSA, B. (2003). L'alternance en formation professionnelle au Québec: du dispositif institutionnel à la pratique des acteurs. Dans M. Hardy (dir.), Concertation éducation-travail: politiques et expériences (p. 187-209). Québec: Presses de l'Université du Québec.

MAZALON, É., BEAUCHER, C. et LANGLOIS, M. (2010). L'évolution et la détermination des projets d'élèves inscrits dans un programme de formation professionnelle en alternance travail-études. Revue de recherche appliquée sur l'apprentissage, 3(10), 1-20.

MINISTÈRE DE L'ÉDUCATION, DU LOISIR ET DU SPORT (2006). L'alternance travail-études en formation professionnelle et technique: c'est plus que des études! Québec: Gouvernement du Québec. Récupéré de www.inforoutefpt.org/ate

MINISTÈRE DE L'ÉDUCATION, DU LOISIR ET DU SPORT (2010). Guide pratique $d u$ superviseur de stage en ATE en entreprise. Guider la connaissance. Québec: Gouvernement du Québec. Récupéré de www.aquaet.qc.ca

PASQUIER, L. (1998). L'accueil d'un jeune en entreprise, quel tutorat? Dans B. Charlot et D. Glasman (dir.), Les jeunes, l'insertion, l'emploi (p. 110-118). Paris: Presses universitaires de France.

SAVOYANT, A. (1995). Guidage de l'activité et développement des compétences dans une entreprise d'insertion. Éducation permanente, 123, 91-100. 